\title{
Isolation of the biofilm producing bacteria from osteomyelitis patients at Hospital Tengku Ampuan Afzan (HTAA), Kuantan
}

\author{
Khairul Ikhwan Yaakob1, Nazri Mohd Yusof ${ }^{2}$, Farahidah Mohamed ${ }^{3,4,5}$, Mohd Affendi Mohd Shafri ${ }^{1,4}$ \\ ${ }^{1}$ Department of Biomedical Science, Kuliyyah of Allied Health Sciences, International Islamic University Malaysia, 25200 \\ Kuantan, Pahang, Malaysia. \\ ${ }^{2}$ Department of Orthopedics Traumatology and Rehabilitation, Kulliyyah of Medicine, International Islamic University \\ Malaysia, 25200 Kuantan, Pahang, Malaysia. \\ ${ }^{3}$ Department of Pharmaceutical Technology, Kuliyyah of Pharmacy, International Islamic University Malaysia, 25200 \\ Kuantan, Pahang, Malaysia. \\ ${ }^{4}$ Institute of Halal Research and Training, International Islamic University, Gombak, Selangor, Malaysia. \\ ${ }^{5}$ iKOPSdn. Bhd., International Islamic University Malaysia, 25200 Kuantan, Pahang, Malaysia. \\ Email: affendishafri@iium.edu.my
}

Received 14 December 2014; Received in revised form 1 April 2015; Accepted 15 April 2015

\begin{abstract}
Aims: Accurate diagnosis and proper treatments of osteomyelitis are often difficult and ineffective due to several reasons such as less sensitive sample collected and the formation of biofilm following prosthetic use. Thus, our goal of this study is to identify suitable sample for laboratory diagnosis and also microbial species that cause osteomyelitic infection and discriminate between biofilm and non-biofilm producing strains in patients at Hospital Tengku Ampuan Afzan, Kuantan.

Methodology and results: Samples of bone, prosthetic material, tissue and swab were collected from patient with suspected osteomyelitis at the hospital. Bacteria were isolated from sample using methods such as homogenization, direct transfer, and sonication. Then, species identification was done by colony characterization, biochemical test and the API identification system. Once species identified, tissue culture plate method was performed to discriminate the biofilm-producing strain from the non-biofilm-producing strain. The total number of 57 samples were collected from 17 cases of suspected osteomyelitis with 34 samples were found positive bacterial growth. Prosthetic samples produced highest positive growth with $81.3 \%$, following by bone samples with $66.7 \%$ while swab and tissue samples with $46.2 \%$ and $43.8 \%$ respectively. We found that 14 from total 16 pathogens identified were biofilm producing-strains.

Conclusion, significance, and impact of study: Prosthetic and bone samples produced higher bacterial growth, in contrast to other type of samples. Sonication method improves bacterial detection. Biofilm producing-bacteria were also the most common isolated strains from osteomyelitic infection. These have underscored the need to revise current clinical and laboratory practice as proper identification biofilm bacteria may influences management an outcome.
\end{abstract}

Keywords: Osteomyelitis; Biofilm; Prosthetic; Sonication.

\section{INTRODUCTION}

Osteomyelitis results from inflammation of the bone caused by infection of pathogenic organisms. It may progress into a chronic and persistent state, leading into necrosis, destruction of bone and interruption in the formation of new bone, which will increase the risk of pathological fracture (Carek et al., 2001). Osteomyelitis can be classified into acute and chronic state of osteomyelitis. Acute osteomyelitis developed within 2 weeks after onset of infection and predominantly occurs in children (Lew and Waldvogel, 2004). On the other hand, chronic osteomyelitis is a persistent, prolonged infection from several months up to years. Unlike acute osteomyelitis, chronic osteomyelitis is more common in adults (Carek et al., 2001).

The most common pathogen in osteomyelitis is Staphylococcus aureus, followed by other species such as Pseudomonas aeruginosa, and Enterobacteriaceae spp. and $90 \%$ of osteomyelitis associated with implant are caused by Staphylococcus epidermidis (Ciampolini and Harding, 2000). It was thought that monoculture of bacteria is commonly associated with non-prosthetic samples such as tissue (Gristina and Costerton, 1985; Thomaidis et al., 2013). Polymicrobial infection of mixed gram positive and gram negative bacteria occurs most 
commonly in patients with certain conditions, notably diabetic foot infection (Ciampolini and Harding, 2000). Different profile of infection is also seen with a different age-group. In the newborn and infant, most bacteria isolated are S.aureus, Streptoccoccus agalacticae, $S$. pyogenes, Escherichia coli, and Haemophilus influenza predominate in children (Brady et al., 2008). Among adult, the most common microorganism causing osteomyelitis is $S$. aureus. Other bacteria that are less frequently found include Enterococcus sp., Streptococcus sp., Enterobacter sp., Mycobaterium sp. and Candida sp. (Calhoun et al., 2009).

Biofilm is a community or an aggregation of interacting bacteria attached to a solid surface or to each other and encased in an exopolysaccharide matrix. This formation plays an important role in the osteomyelitis giving rise to persistent infection that lead to the chronic state. Bacterial film can comprise of single species or multiple microbial species congregating to form biofilm on a range of biotic and abiotic surfaces (O'Toole et al., 2000) such as the surface of prosthetic material and necrotic bone tissue (Brady et al., 2008). The biofilm that formed have special features and characteristics such as slow penetration of antibiotic through the biofilm matrix, changes in the growth rate of biofilm bacteria, and physiological alteration due to the biofilm mode of growth that cause ineffective treatment with antimicrobial agents (Donlan and Costerton, 2002), raising the concern of treating nondiscriminated osteomyelitis with common or widespectrum antimicrobial agents. Different species have different virulence factors and required specific antimicrobial treatments. Inability to identify the accurate species and biofilm-producing strain lead to false positive and false negative diagnosis that will cause wrong treatment management. By identifying biofilm formation ability; we can create the proper treatment management plan that can effectively eradicate the biofilm.

Osteomyelitis diagnosis is often based on clinical findings, including information from medical history, physical examination and laboratory tests. However the benchmark and important measures of osteomyelitis are based on the laboratory tests. The microbiology examination that is included in the laboratory tests is the gold standard for this diagnosis (Carek et al., 2001). An accurate organism identification result depends on the type of specimen such as bone, tissue, and prosthetic implants (Larsen et al., 2012). Other specimen such as sinus tract is not reliable. Sometimes previous antibiotic administration may interrupt the diagnostic accuracy (Legout and Senneville, 2013).

Thus, in this present study, we identify suitable sample for laboratory diagnosis and also microbial species that cause osteomyelitic infection and discriminate between biofilm and non-biofilm producing strains in patients at Hospital Tengku Ampuan Afzan, Kuantan.

The modified criteria that are used in this study are; (1) positive organism cultured from bone, (2) evidence of osteomyelitis on direct examination during surgery or histopathologic examination and (3) at least 2 signs of symptom such as fever, localized swelling, tenderness, heat, or drainage at suspected site of bone infection. These criteria are based on Centre of Disease Control (CDC)'s criteria for osteomyelitis infection (ECDC, 2012).

The present study would like to highlight the importance of discriminating type of samples, sonication method and biofilms strains. The accurate identification and accurate diagnosis will result in the proper treatment for osteomyelitis.

\section{MATERIALS AND METHODS}

\section{Sample collection}

All samples were collected from patients with suspected osteomyelitis infection during surgery at the Orthopaedic Operation Theatre Hospital Tengku Ampuan Afzan from September 2013 until January 2014. Diagnosis was made based on the Centre of Control Disease (CDC)'s (as mentioned earlier in the introduction) (ECDC, 2012). The sample collected once the patient read and signed a consent form. Samples were stored in suitable containers at temperature $2-8^{\circ} \mathrm{C}$ and transported to the Microbiology Research Laboratory, Kuliyyah of Allied Health Science, IIUM Kuantan. Samples were processed within $48 \mathrm{~h}$ of collection.

\section{Bacterial Isolation}

\section{Swab sample}

Swab samples were obtained using Amies Transport Medium with Charcoal (Healy and Freedman 2006). In the laboratory, each swab sample was spread directly on the agar media and incubated at $37^{\circ} \mathrm{C}$ for $24 \mathrm{~h}$ (Figure 1).

\section{Tissue sample}

Tissue samples were homogenized by shaking them with $5 \mathrm{~mL}$ phosphate buffer solution (PBS) (pH 7.2) and 10 ballotini beads in accordance to tissue size at $250 \mathrm{rpm}$ for $10 \mathrm{~min}$ as described by Patridge and Towsend (2012). After homogenization, $100 \mu \mathrm{L}$ of solution was transferred and spread onto the agar media. Plates were then incubated for $24 \mathrm{~h}$ at $37^{\circ} \mathrm{C}$ (Figure 1).

\section{Bone, prosthetic and beads}

Bone and prosthetic samples were processed with the sonication method as described by Trampuz et al. (2007). PBS $(\mathrm{pH}$ 7.2) was added into each container until the sample was fully submerged in the solution. The sample were later shaken at $250 \mathrm{rpm}$ for $10 \mathrm{~min}$ and subjected to sonication in the ultrasonic water bath (Dentsply Neytech, USA) at a frequency of $44 \pm 2 \mathrm{kHz}$ for $5 \mathrm{~min}$. The process was repeated for two times and $100 \mu \mathrm{L}$ of sonicated fluid was spread on the agar media and incubated for $24 \mathrm{~h}$ at $37^{\circ} \mathrm{C}$ (Figure 1 ). 


\section{Bacterial identification}

After $24 \mathrm{~h}$ of incubation, the colonies from each agar media (blood agar, tryptic soy agar, MacConkey agar) were subjected to identification assays to determine species by colony characteristics, biochemical test, growth on selective agar media (mannitol salt agar, cetrimide agar) and API Identification System (Biomerieux, France). Plates with low numbers of colony were incubated for another $24 \mathrm{~h}$ at the same temperature. Plates that were completely lacked in bacterial growth after $48 \mathrm{~h}$ of incubation were classified as no growth plates. As biofilmproducing strain is the only one that is able to adhere to solid surface, positive growth from either prosthetic or bone sample from each patient was selected. They are subjected for species identification and biofilm detection. Bone and prosthetic samples are solid type of material that possesses high chances of biofilm growth compared to tissue and swab samples (Brady et al., 2006; Larsen et al., 2012)

\section{Biofilm detection}

The bacterial biofilm assessment was performed using the Tissue Culture Plate method for biofilm detection as described by Stepanović et al. (2007) with some modifications. The test strains from stock cultures were prepared as working cultures from overnight incubation on the tryptic soy agar (TSA). After verifying the strain purity, 3 to 4 identical colonies were suspended in $5 \mathrm{~mL}$ tryptic soy broth (TSB) and incubated for $18 \mathrm{~h}$ at $37^{\circ} \mathrm{C}$. After incubation, the cultures in TSB were vortexed and diluted in 1:100 Tryptic soy broth supplemented with 1\% glucose (TSBg). The diluted bacteria were vortexed and transferred into a tissue culture plate with flat bottom (Corning, USA) and incubated for $24 \mathrm{~h}$ at $37^{\circ} \mathrm{C}$. The contents of incubated tissue culture plates were decanted. Each well was washed three times with a 300 $\mu \mathrm{L}$ PBS (pH 7.2). After washing, the plates were dried at room temperature before fixation. Then, the biofilm in the well were fixed with $150 \mu \mathrm{L}$ methanol for $20 \mathrm{~min}$ and left to air dried overnight in an inverted position at room temperature. The dried plates later were stained with 150 $\mu \mathrm{L}$ of $1 \%$ crystal violet for $15 \mathrm{~min}$. After staining, the plates were washed with distilled water and dried. Then the crystal violets were resolubilized with $150 \mu \mathrm{L}$ of $95 \%$ ethanol. Optical density (OD) reading was taken for assessment. Wells with OD reading more than ODc (average OD of negative control +3 (SD of negative control) were assumed as indicative of biofilm production (Figure 2).

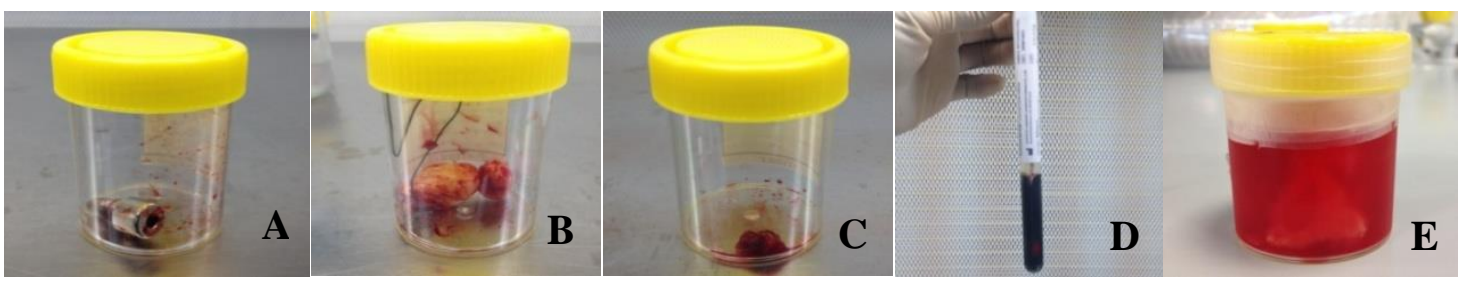

Figure 1: Type of samples A, prosthetic implant; B, antibiotic beads; C, tissue; D, swab; and E, bone

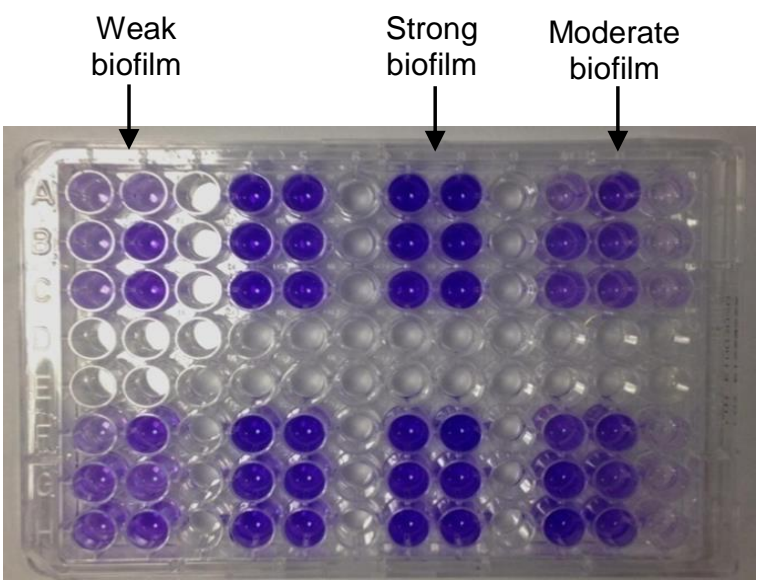

Figure 2: Tissue Culture Plate method for biofilm detection showing strength of biofilm production indicated by colour intensity with crystal violet. Strong purple indicates high biofilm production and weak purple indicates low biofilm production.

\section{RESULTS}

Since September 2013 until July 2014, total number of samples taken was 57 from 17 cases (Patient ID OS001OS017) of orthopaedic surgery with suspected osteomyelitis at Hopital Tengku Ampuan Afzan, Kuantan. Total sample that found positive growth was 34 which were $86.7 \%$ prosthetic samples, $66.7 \%$ Bone samples, $46.2 \%$ Swab samples and $43.8 \%$ Tissue samples (Table $1)$. These samples with bacterial growth were then identified as coming from only 14 patients, two of which were also polymicrobial in character.

It was also found that the most common pathogen identified was $S$. aureus $(n=10)$, followed by $S$. epidermidis $(n=3)$ and $P$. aeruginosa $(n=3)$ (Table 2). From total ten, nine $S$. aureus isolated were biofilm producing strains with five being strong producers, two moderate and two weak producers. For $S$. epidermidis, two were biofilm producer with one being a strong and one being a moderate biofilm producer. All three $P$. aeruginosa isolated were moderate biofilm producer (Table 2). 
Table 1: Number of samples with bacterial growth.

\begin{tabular}{ccccc}
\hline Sample & Collected & Bacterial growth & Mono growth & Poly growth \\
\hline Bone & 12 & $8(66.7 \%)$ & 6 & 2 \\
Prosthesis & 15 & $13(86.7 \%)$ & 9 & 4 \\
Tissue & 16 & $7(43.8 \%)$ & 5 & 2 \\
Swab & 13 & $6(46.2 \%)$ & 4 & 2 \\
TOTAL & 57 & $34(59.6 \%)$ & 24 & 10 \\
\hline
\end{tabular}

Table 2: Species of bacteria isolated from each patients.

\begin{tabular}{ccc}
\hline Patient ID & Species isolated & Biofilm formation \\
\hline OS001 & S. aureus & +++ \\
OS002 & S. epidermidis & +++ \\
OS003 & S. epidermidis & - \\
& S. aureus & ++ \\
OS004 & S. aureus (Small Colony Variant) & - \\
OS005 & P. aeruginosa & + \\
OS006 & S. aureus & - \\
OS007 & No growth & ++ \\
OS008 & S.epidermidis & - \\
OS009 & No growth & ++ \\
OS010 & P. aeruginosa & + \\
OS011 & S. aureus & ++ \\
OS012 & P. aeruginosa & - \\
OS013 & No growth & ++ \\
OS014 & S. aureus & +++ \\
OS015 & S. aureus & +++ \\
OS016 & S. aureus & ++ \\
OS017 & S. aureus & ++ \\
\hline
\end{tabular}

+++, High biofilm; ++, Moderate biofilm; +, Low biofilm; - , None biofilm producer

\section{DISCUSSION}

The purpose of this study is to describe the profile of main bacteria which cause osteomyelitis in Kuantan, Pahang and look into the frequency of the biofilm microorganism. The differences arising from variation in samples, isolated using sonication method, are also characterized. These are done in the background of some limitations namely the short duration of the study and the limited number of source of samples (i.e. from only one hospital). Besides, the study does not look into age, racial, gender and financial/social status differences among the patients. The results are thus non-representative for Pahang or Malaysia but would still be useful for expanding the view of current issues involving osteomyelitis and prosthetic joint infection (PJI).

\section{The role of S. epidermidis}

The highest frequency of isolated bacteria is S.aureus. This is not surprising as this microorganism is already established as the leading pathogen in osteomyelitic infection (Lew and Waldvogel 2004). Other species found were $P$. aeruginosa and $S$. epidermidis. Most osteomyelitis are related to secondary infection and nosocomial infection; both $S$. aureus and $P$. aeruginosa are already known worldwide to be common in these types of infections (Hughes et al., 2005). However, S. epidermidis (coagulase negative staphylococci) is not well-established as an osteomyelitis-causing pathogen. This may be the major reason why it is normally not discriminated in microbiology laboratory as coagulasenegative are considered as microorganism with little virulence and usually as contaminant (Aragón-Sánchez et al., 2010)

In the study, 3 out of 16 identified species (19\%) were S.epidermidis infection; 2 out of these 3 are biofilm producer and both are gentamicin-resistant. Bacteria other than $S$. aureus and $P$. aeruginosa have been suspected of having a more central role in inflammatory conditions and osteomyelitis. Here, we also call for an increase in the attention given to $S$. epidermidis and a longer, and more sampling to affect changes at management and clinical lab level - such as change in the practice where polymixed growth should be further discriminated.

Staphylococcus epidermidis has been described as a significant emerging pathogen in osteomyelitic infection especially in implant-related infection. O'Gara and 
Humphreys (2001), Ziebuhr et al. (2006), and Valour et al. (2013), described S. epidermidis infection as an infection which is not as invasive as $S$. aureus. In contrast to $S$. aureus and $P$. aeruginosa that can be virulence in both planktonic or biofilm forms, all infections associated with $S$. epidermidis are only ascribed to when the pathogen achieve the capability to form biofilm. According to Aragón-Sánchez et al. (2010) the outcomes of surgical treatments are similar in both $S$. epidermidis and $S$. aureus infection. This observation however may not be similar if the treatment is based on antibiotic administration only, especially if the infected pathogens have developed resistant against antimicrobial agents. It is thus imperative to develop a method where $S$. epidermidis infection and its clinical repercussion could be analysed to provide an accurate diagnosis and deliver better treatment plan especially in terms of antibiotic and debridement surgery.

\section{Biofilm-producing bacteria}

More than $3 / 4$ of the bacteria isolated were biofilm producers. Biofilm are the attachment of microorganism communities and formation of exopolysaccharide matrix on the solid surface. Most treatments for osteomyelitis have failed or become ineffective due to the development of biofilm at the site of infection (Brady et al., 2006). There are several biofilm-related mechanisms that lead to antibiotic resistance such as the role of the biofilm matrix forming a barrier for antibiotic penetration, slow growth of the biofilm due to nutrient limitation, activation of the general stress response and activation of specific biofilm phenotype (Mah and O'Toole, 2001). Just as it is important to detect the specific type of bacteria involved, it is also imperative to discriminate the difference in strains' ability to produce biofilm as this may be reflected in the difference in clinical outcome and management.

In this regard, a change in current hospital lab policy may be needed. The results of the study contrasts with the current prevailing assumption that non prosthetic sample only possess monogrowth compared to polygrowth on prosthetic samples. This assumption may have been based on previous findings by the experts of the time such as the report by Gristina et al. (1985). Here, we found that 6 out of $10(60 \%)$ polygrowths came from non-prosthetic samples (tissue, bone and swab). The type of bacterial species isolated from prosthetic samples is always the same species found in non-prosthetic samples. Also, the absence of culture in prosthetic samples will also produce no positive in non-prosthetic samples.

\section{Importance of prosthetic sample}

Malaysian medical microbiology laboratories (bacteriology) currently do not accept prosthetic implant as a sample. This may lead to cases where a negative culture from non-prosthetic sample will lead to false negative situation, in which an existing pathogen may not be recognized as a causing agent. The implication of this is that a generalized treatment may be given, when a more specific treatment tailored to individual cases could have been offered. The prosthetic samples may be the only place where infective agent, in general, and biofilmproducing agent in specific, may be found.

In this study, all prosthetic samples have been shown to have positive bacteria growth. This indicates the importance to include prosthetic material as a sample for laboratory in the diagnosis of osteomyelitis especially when implant related infections occur and when prosthetic sample is available. Previous studies by Trampuz et al. (2007), Esteban et al. (2008) and Piper et al. (2009) have shownthat prosthetic materials increased sensitivity in isolation of biofilm-producing bacteria.

The biofilm-producing bacteria have been known to be capable to transform from the planktonic to the complex biofilm form, and of recruiting non-adherence bacteria to the biofilm matrix. The mechanism involve may be through gene transfer or quorum sensing mechanism (Sakuragi and Kolter, 2007). As biofilm can only occur on solid surface material, no bacteria can be obtained from tissue sample if entire planktonic bacteria cease to occur. Thus, in theory, if tissue sample is the only sample processed and the prosthetic sample is discarded or ignored, this could cause false negative results and lead to less specific treatment.

\section{The role of sonication method}

Isolation of biofilm bacteria especially from prosthetic samples was deemed difficult. A good method with optimization is therefore crucial. We have done sample processing with and without sonication (Figures 3 and 4) and find that without sonication method, the plate showed less or no bacteria colony growth. However, after sonication method, there were significant numbers of colonies growth. Therefore we concluded that the use of sonication method as a processing method for bone and prosthetic sample improved bacterial isolation. The sonication method employed ultrasound at a frequency between $20-40 \mathrm{kHz}$ that is able to remove adherent microorganisms from prosthetic and bone samples (Trampuz et al., 2007; Piper et al., 2009; Larsen et al., 2012). Other non-culture diagnostic methods such as immunofluorescene microscope (IFM), and polymerase chain reaction (PCR), exist and has been shown to have high sensitivity, but determination of antimicrobial sensitivity is not possible due to the non-culture technique in use (Achermann et al., 2010). As sensitivity testing is important for proper treatment plans with antibiotic, these methods are not the method of choice for diagnosis or for practical consideration.

\section{CONCLUSION}

From this short study, we have found that prosthetic samples and bone samples were more sensitive than other type of sample and also biofilm producing bacteria were the most common causes in osteomyelitic infection. Successful treatment of osteomyelitis depends on a proper identification of the species and strain of pathogen 


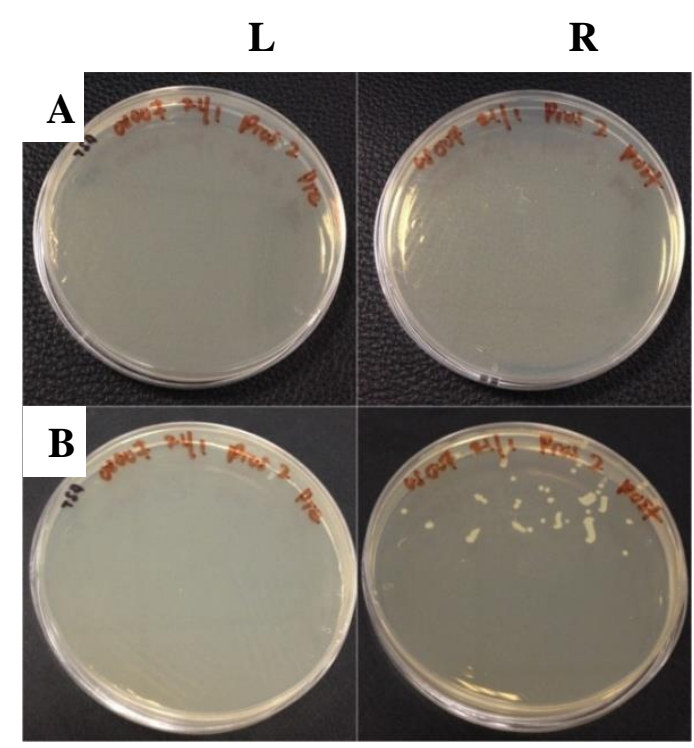

Figure 3: Bacterial culture showing colony isolated from prosthetic sample with (A) denotes culture at $24 \mathrm{~h}$ incubation and $(B)$ denotes culture at $48 \mathrm{~h}$ incubation. The left-hand sides (L) were cultured in the absence of sonication and showed no bacterial growth. The righthand side $(R)$ were cultured after sonication and showed colony growth.

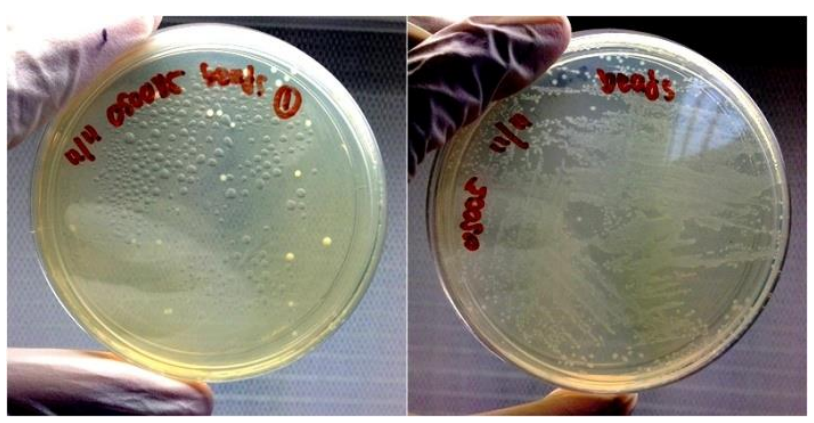

Figure 4: Colony isolated from antibiotic beads at $24 \mathrm{~h}$ incubation period. The left-hand side $(\mathrm{L})$ was grown in the absence of sonication and showed few colony growths (small, white colonies). The right-hand side $(R)$ was grown after sonication and showed more bacterial growth.

that cause the infection. To get the accurate diagnosis and the best treatment towards patient care, proper sampling with a good diagnostic method such as sonication methods and identification needs to be considered for inclusion in current hospital lab practice.

\section{ACKNOWLEDGEMENT}

This study was supported by research funds from Ministry of Higher Education Malaysia (MOHE) through
Fundamental Research Grant Scheme (FRGS) No. FRGS13-041-0282.

\section{REFERENCES}

Achermann, Y., Vogt, M., Leimig, M., Wüst, J. and Trampuz, A. (2010). Improved diagnosis of periprosthetic joint infection by Multiplex PCR of sonication fluid from removed implants. Journal of Clinical Microbiology 48(4), 1208-1214.

Aragón-Sánchez, J., Lázaro-Martínez, J. L., Hernández-Herrero, M. J., Quintana-Marrero, Y. and Cabrera-Galván, J. J. (2010). Clinical significance of the isolation of Staphylococcus epidermidis from bone biopsy in diabetic foot osteomyelitis. Diabetic Foot and Ankle 1, 1-5.

Brady, R. A., Leid, J. G., Corteston, J. W. and Shirtliff, M. E. (2008). Osteomyelitis and the role of biofilms in chronic infection. FEMS Immunology and Medical Microbiology 52(1), 13-22.

Brady, R. A., Leid, J. G., Corteston, J. W. and Shirtliff, M. E. (2006). Osteomyelitis: Clinical overview and mechanisms of infection. Clinical Microbiology Newsletter 28(9), 65-72.

Calhoun, J., Manring, M. and Shirtliff, M. (2009). Osteomyelitis of the long bones. Seminars in Plastic Surgery 1(212), 59-72.

Carek, P., Dickerson, L. and Sack, J. (2001). Diagnosis and management of osteomyelitis. American Family Physician 84(9), 1027-1033.

Ciampolini, J. and Harding, K. G. (2000). Pathophysiology of chronic bacterial osteomyelitis. Why do antibiotics fail so often? Postgraduate Medical Journal 76(898), 479-483.

Donlan, R. and Costerton, J. (2002). Biofilms: Survival mechanisms of clinically relevant microorganisms. Clinical Microbiology Reviews 15(2),167-193.

Esteban, J., Gomez-Barrena, E., Cordero, J., Martinde-Hijas, N. Z., Kinnari, T. J. and Fernandez-Roblas, R. (2008). Evaluation of quantitative analysis of cultures from sonicated retrieved orthopedic implants in diagnosis of orthopedic infection. Journal of Clinical Microbiology 46(2), 488-492.

ECDC, European Centre For Disease Prevention and Control, (2012). Point prevalence survey of healthcare-associated infections and antimicrobial use in European acute care hospitals - Protocol 4.3. Stockholm: ECDC. pp 49.

Gristina, A. G. and Costerton, J. W. (1985). Bacterial adherence to biomaterials and tissue. The significance of its role in clinical sepsis. The Journal of Bone and Joint Surgery (American Volume) 67(2), 264-273.

Healy, B. and Freedman, A. (2006). Infections. British Medical Journal 332,838-841.

Hughes, A. J., Ariffin, N., Huat, T. L., Abdul-Molok, H., Hashim, S., Sarijo, J., Abd-Latif, N. H., Abu-Hanifah, Y. and Kamarulzaman A. (2005). Prevalence of nosocomial infection and antibiotic use at a university medical center in Malaysia. Infection Control and Hospital Epidemiology 26(1), 100-104. 
Larsen, L. H., Lange, J., Xu, Y. and Schonheyder, H. C. (2012). Optimizing culture methods for diagnosis of prosthetic joint infections: A summary of modifications and improvements reported since 1995. Journal of Medical Microbiology 61(3),309-316.

Legout, L. and Senneville, E. (2013). Periprosthetic joint infections: Clinical and bench research. The Scientific World Journal 2013,12-14.

Lew, D. P. and Waldvogel, F. A. (2004). Osteomyelitis. Lancet 364(9431), 369-379.

Mah, T. F. and O'Toole, G. A. (2001). Mechanisms of biofilm resistance to antimicrobial agents. Trends in Microbiology 9(1), 34-39.

O'Gara, J. P. and Humphreys, H. (2001). Staphylococcus epidermidis biofilms: Importance and implications. Journal of Medical Microbiology 50(7), 582-587.

O'Toole, G., Kaplan, H. B. and Kolter, R. (2000). Biofilm formation as microbial development. Annual Review of Microbiology 54, 49-79.

Patridge, D. G. and Towsend, R. (2012). Microbiological processing of samples in the investigative of suspected prosthetic joint infection. In: Infected Total Joint Arthroplasty - The Algorithmic Approach.Trebse, R. (ed.). Springer New York. pp. 181-193.

Piper, K. E., Jacobson, M. J., Cofield, R. H., Sperling, J. W., Sanchez-Sotelo, J., Osmon, D. R., McDowell, A., Patrick, S., Steckelberg, J. M., Mandrekar, J. N., Fernandez-Sampredo, M. and Patel, R. (2009). Microbiologic diagnosis of prosthetic shoulder infection by use of implant sonication. Journal of Clinical Microbiology 47(6), 1878-1884.

Sakuragi, Y. and Kolter, R. (2007). Quorum-sensing regulation of the biofilm matrix genes (pel) of Pseudomonas aeruginosa. Journal of Bacteriology 189(14), 5383-5386.

Stepanović, S., Vuković, D. and Hola, V. (2007). Quantification of biofilm in microtiter plates: Overview of testing conditions and practical recommendations for assessment of biofilm production by staphylococci. Acta Pathologica, Microbiologica, et Immunologica Scandinavica 115(8), 891-899.

Thomaidis, P. C., Vlamis, J., Evangelopoulos, D. and Stylianakis, A. (2013). The impact of sonication on the microbiological diagnosis of the orthopedic periprosthetic infections. Acta Orthopaedica et Traumatologica Hellenica 64(3), 146-150.

Trampuz, A., Piper, K. E., Jacobson, M. J., Hanssen, A. D., Unni, K. K., Osmon, D. R., Mandrekar, J. N., Cockerill, R., Steckelberg, J. M., Greenleaf, J. F. and Patel, R. (2007). Sonication of removed hip and knee prostheses for diagnosis of infection. The New England Journal of Medicine 357(7), 654-663.

Valour, F., Trouillet-Assant, S., Rasigade, J. P., Lustig, S., Chanard, E., Meugnie, H., Sylvestre, T., Vandenesch, F., Etienne, J., Ferry, T. and Laurent, F. (2013). Staphylococcus epidermidis in orthopedic device infections: The role of bacterial internalization in human osteoblasts and biofilm formation. PloS ONE 8(6), 1-11.
Ziebuhr, W., Hennig, S., Eckart, M., Kränzler, H., Batzilla, C. and Kozitskaya, S. (2006). Nosocomial infections by Staphylococcus epidermidis: How a commensal bacterium turns into a pathogen. International Journal of Antimicrobial Agents 28(1), S14-S20. 